\title{
A Relação Professor-Aluno no Ensino Médio: Percepção do Professor de Escola Pública
}

\author{
Victor Silva Ferreira \\ Faculdades Integradas Campos Sales - São Paulo - SP - Brasil \\ Márcia Siqueira Andrade \\ Centro Universitário UNIFIEO - Osasco - SP - Brasil
}

\begin{abstract}
Resumo
Esta pesquisa buscou identificar a percepção dos professores quanto à relação professor-aluno a partir de indicadores de conflito e afinidade, relacionando-os com as variáveis desempenho acadêmico, idade, sexo dos alunos e dos docentes. Realizou-se pesquisa transversal de caráter correlacional, com 10 professoras do ensino médio de uma escola pública estadual, que avaliaram 10 alunos utilizando a Escala da Relação Professor-Aluno (ERPA). Os resultados sugerem que as professoras tendem a ter maior afinidade com alunas e relação conflituosa com os discentes do sexo masculino. Ao correlacionar a variável de desempenho acadêmico com os indicadores de afinidade e conflito verificou se que existe correlação positiva fraca entre o indicador de conflito e o desempenho acadêmico da disciplina Filosofia. Contudo, na disciplina de Geografia, existe também uma correlação positiva fraca, mas com o indicador de afinidade. Conclui-se pela necessidade de novos estudos que aprofundem o tema abordado considerando outros contextos.
\end{abstract}

Palavras-chave: Interação professor-aluno; conflito; atração interpessoal.

\section{The Teacher-Student Relationship in High School: Perception of the Public School Teachers}

\begin{abstract}
This research aimed to identify the teachers' perception of the teacher-student relationship from conflict and affinity indicators, relating them to the variables academic performance, age, gender of students and teachers. Transversal cross-sectional research was carried out with 10 high school teachers from a state public school, who evaluated 10 students using the Teacher-Student Relationship Scale (ERPA). The results suggest that teachers tend to have greater affinity with female students and a conflicting relationship with male students. When correlating the academic performance variable with the affinity and conflict indicators, it was verified that there is a weak positive correlation between the conflict indicator and the academic performance of the discipline Philosophy. However, in the Geography discipline, there is also a weak positive correlation, but with the affinity indicator. It was concluded that new studies are needed to deepen the theme addressed in other contexts.
\end{abstract}

Palavras-chave: Teacher student interaction; conflict; interpersonal attraction.

\section{La relación profesor-alumno en la enseñanza secundaria: percepción del profesor de escuela pública}

\section{Resumen}

En esta investigación se buscó identificar la percepción de los profesores en lo que se refiere a la relación profesor-alumno a partir de indicadores de conflicto y afinidad, relacionándolos con las variables desempeño académico, edad, sexo de los alumnos y de los docentes. Se realizó investigación transversal de carácter correlacional, con 10 profesores de la enseñanza secundaria de escuela pública estadual, que evaluaron 10 alumnos utilizando la Escala de la Relación Profesor-Alumno (ERPA). Los resultados sugieren que las profesoras tienden a tener más afinidad con alumnas y relación de conflictos con los discentes del sexo masculino. Al correlacionar la variable de desempeño académico con los indicadores de afinidad y conflicto se verificó que hay correlación positiva escasa entre el indicador de conflicto y el desempeño académico de la asignatura Filosofía. Pero, en la asignatura de Geografía, hay también una correlación positiva escasa, pero con el indicador de afinidad. Se concluye por la necesidad de nuevos estudios que profundicen el tema abordado considerando otros contextos.

Palabras clave: Interación profesor-alumno; conflicto; atracción interpersonal. 


\section{Introdução}

O estudo desta pesquisa é a relação professor aluno associado aos indicadores de conflito que se refere às características negativas nessas interações, como imprevisibilidade, discordância e desprazer e a afinidade, sendo os aspectos positivos, como abertura, afeto e segurança (Petrucci, Borsa, Barbosa, \& Koller, 2014), onde o relacionamento professor-aluno exerce um papel importante no desenvolvimento cognitivo, social e emocional dos discentes, influenciando não apenas o desempenho escolar, mas também o funcionamento social e emocional (Hamre \& Pianta, 2001; Myers \& Pianta, 2008; Pianta, 1999). Dessa forma, alunos que apresentam relacionamentos de alta qualidade com seus docentes têm melhor desempenho escolar (Berry \& O'Connor, 2010; Maldonado-Carreño \& Votruba-Drzal, 2011; Pianta \& Stuhlman, 2004).

Com isso, as diversas etapas do ciclo escolar apresentam desafios que requerem habilidades específicas dos alunos para conseguirem superá-las. O relacionamento positivo com os professores pode facilitar o processo de adaptação dos estudantes contribuindo para a aprendizagem de habilidades essenciais para a trajetória escolar e para a redução de problemas de comportamento atuais e futuros (O'Connor, Collins, \& Supplee, 2012; Pianta \& Stuhlman, 2004; Silver, Measelle, Armstrong, \& Essex, 2005).

Além disso, embora os interesses dos alunos se modifiquem ao longo do tempo, as interações com os professores parecem continuar influenciando o seu desenvolvimento até as séries finais da trajetória escolar (Crosnoe, Johnson, \& Elder, 2004; Hamre \& Pianta, 2006; Pianta \& Allen, 2008).

Diante da importância desses relacionamentos para o funcionamento social, emocional e cognitivo dos alunos, destaca-se a necessidade de investigações que visem compreender aspectos relativos ao relacionamento professor-aluno no contexto brasileiro, mediante a pergunta: qual a percepção dos professores sobre a qualidade do seu relacionamento com os alunos? Dessa forma, procura se elucidar a pergunta do referido estudo, de modo que características do relacionamento professor-aluno podem não ser generalizáveis para outras populações, embora sua relevância para o desenvolvimento dos alunos seja inquestionável (Petrucci, Borsa, Barbosa, \& Koller, 2014).

Assim, a hipótese deste estudo é baseada na percepção dos professores quanto ao seu relacionamento com os alunos do ensino médio está associada às variáveis de desempenho acadêmico, idade, sexo dos discentes e sexo dos respondentes.

Mediante o exposto o objetivo desta pesquisa é identificar a percepção dos professores quanto à relação professor-aluno a partir de indicadores de conflito e afinidade, relacionando-os às variáveis de desempenho acadêmico, idade e sexo dos alunos e sexo dos respondentes.

\section{Método}

A pesquisa é transversal de caráter quantitativo correlacional, pois busca elucidar a relação das variáveis dependentes: conflito e afinidade, com as variáveis independentes: desempenho acadêmico, idade, sexo dos alunos e sexo dos docentes, através da correlação desses dados.

\section{Participantes}

Participaram deste estudo 10 professores que lecionavam em salas do primeiro ao terceiro ano do ensino médio, onde todos têm formação em pedagogia e foram selecionados por conveniência. Além disso, cada docente era responsável por sua turma há dez meses. Para possibilitar a existência de familiaridade, esse critério foi adotado entre cada professor (a) e seus respectivos alunos.

Os docentes que participaram da pesquisa foram sete professores, sendo dois de filosofia, dois de física, um de sociologia, um de educação física e um de história, e três professoras sendo uma de português, uma de geografia e uma de história, totalizando 10 docentes; as idades variaram de 38 a 54 anos $(M=44,80 ; \mathrm{DP}=5,39)$.

Os alunos possuíam a idade entre 16 e 19 anos, sendo 47 do sexo masculino e 53 do sexo feminino, totalizando 100 alunos $(M=16,51 ; D P=0,82)$. Eles frequentavam turmas do segundo ano do ensino médio.

\section{Campo da pesquisa}

A presente pesquisa foi realizada em uma escola estadual no município de Cotia, no estado de São Paulo-SP, que funciona em três turnos: matutino, vespertino e noturno. No turno matutino estudam os alunos da oitava série até o terceiro grau do ensino médio, no período vespertino as turmas de sexta série até as de oitava série do ensino fundamental e no noturno, os alunos do primeiro ao terceiro ano do ensino médio.

\section{Instrumentos}

O Questionário Socioeconômico foi utilizado para mensurar as características de cada professor que respondeu a pesquisa, em relação ao sexo, idade, estado civil, número de filhos, escolaridade e renda familiar.

O instrumento contendo os Dados dos Alunos Coletados nos Prontuários consiste nos atributos que possuem cada aluno como o sexo, a idade, escolaridade e desempenho acadêmico em português, física, filosofia, sociologia, geografia, educação física e história, sendo este desempenho a média final do ano letivo de 2014

Também foi utilizada a Escala de Relacionamento Professor-Aluno (ERPA), uma versão da Student-Teacher Relationship Scale: Short Form (STRS-SF), sendo utilizados com professores de todas as séries escolares, desde a infância até a adolescência, e adaptada e validada para a população brasileira (Petrucci \& cols., 2014). A ERPA avalia a percepção dos professores sobre a qualidade do seu re- 
lacionamento com cada aluno por meio de indicadores de afinidade e conflito nas interações.

É constituída por 15 itens, em uma escala do tipo $\mathrm{Li}$ kert de cinco pontos para cada item, sendo: (1) sempre, (2) quase sempre, (3) às vezes, (4) quase nunca e (5) nunca. Sete itens $(1,3,5,6,7,9$, 15) representam relações adequadas entre docentes e discentes, que constituem o fator afinidade e oito itens $(2,4,8,10,11,12,13,14)$ refletem interações disfuncionais e compõem o fator conflito (Petrucci \& cols., 2014).

\section{Procedimentos}

A coleta de dados foi organizada em quatro momentos. Atendendo à Resolução 196/96, do Conselho Nacional de Saúde, num primeiro momento foi assinado o Termo de Autorização para a Realização de Entrevistas para o desenvolvimento da pesquisa no espaço institucional, assinado pelo diretor da escola, que concedeu a permissão para aplicar o questionário aos professores dos períodos matutino e noturno do ensino médio. Logo após, em uma segunda situação foram emitidas cartas informando aos professores participantes sobre o tema e o objetivo do estudo, assegurando que as informações serão tratadas anônima e sigilosamente e servirão apenas para fins técnico-científicos, portanto os participantes assinaram um Termo de Consentimento Livre e Esclarecido.

Numa terceira ocasião foram coletadas as informações de seis questões sobre o Perfil Socioeconômico dos Professores que foi entregue simultaneamente com a ERPA (Escala de Relacionamento Professor e Aluno), (Petrucci \& cols., 2014) para o preenchimento, com entrega em data previamente combinada, pois devido ao acesso a cada um dos professores, foi solicitado que respondessem às questões sobre como eles percebiam sua relação com os alunos, levando o questionário para casa e posteriormente foi entregue no prazo de cinco dias, a iniciar da data que o questionário foi entregue em mãos.

E por último, foram coletados os Dados de Desempenho Acadêmico dos Alunos nas fichas cadastrais da escola na própria secretaria da instituição.

\section{Análise dos dados}

Os dados obtidos foram tabulados na planilha Excel e exportados para a planilha do software SPSS (versão 18 portable), o que permitiu obter medidas que foram utilizadas na elaboração das analises estatísticas.

Assim os 10 professores avaliaram a qualidade do seu relacionamento com 10 alunos, totalizando 100 questionários da escala ERPA (Escala de Relacionamento Professor Aluno), (Petrucci \& cols., 2014) que foram selecionados através da sequência numérica do diário de classe, ou seja, os dez primeiros alunos da lista consecutivamente.
Os dados serão tratados quantitativamente, adotando-se um nível de significância de 0,05 por convenção. Foram feitas as análises de índice de precisão (Alpha de Cronbach), correlação ( $r$ de Person) e análise multivariada (Manova).

As análises de convergência foram realizadas por meio de análises de correlação de Pearson entre os resultados da ERPA, respondida pelos professores, e os resultados do desempenho acadêmico, sexo e idade dos alunos coletado dos prontuários dos alunos e do sexo e idade dos professores, coletados do questionário socioeconômico. Também foram feitas análises multivariadas para comparar os resultados na ERPA entre sexo dos alunos e professores e calculado o índice de precisão Alpha de Cronbach, para avaliar a consistência interna dos itens que compõem a escala ERPA.

Tabela 1. Confiabilidade Estatística.

\begin{tabular}{ccc}
\hline Constructos & $\begin{array}{c}\text { Alfa de } \\
\text { Cronbach }\end{array}$ & $\mathrm{N}$ \\
\hline Afinidade & 0,82 & 7 \\
Conflito & 0,78 & 8 \\
\hline
\end{tabular}

Nota. Precisão da Escala (ERPA), N (número de itens da Escala ERPA).

\section{Resultados e Discussão}

O presente estudo apresenta valores adequados do alfa de Cronbach em relação ao instrumento ERPA (Escala de Relação Professor Aluno). Calculou-se a consistência interna da escala, obtendo para os fatores afinidade $(0,82 ; \mathrm{N}=$ 7 ) e conflito $(0,78 ; N=8)$. Comparados a outros estudos, os valores possuem uma proporção próxima, $(0,92)$ para os fatores afinidade e $(0,87)$ para conflito (Petrucci \& cols., 2014).

A versão original do questionário, STRS-SF (Student-TeacherRelationshipScale - Short Form), aponta para os fatores de afinidade $(0,85)$ e $(0,88)$ para conflito (Pianta, 1992). Com isso, pode se considerar que as análises subsequentes são internamente consistentes, pois os resultados dos outros estudos do alfa de Cronbach estão próximos dos resultados apresentados por esta pesquisa.

Para identificar a percepção dos professores quanto ao seu relacionamento com os alunos do ensino médio a partir de indicadores de conflito e afinidade foram efetuadas as análises descritiva e multivariada (Manova).

Comprova-se que as alunas tendem a ter mais afinidade (Média= 23,23) do que conflito (Média= 17,57) com as professoras. Em seguida, a análise multivariada, apresentou resultados estatisticamente significativos para afinidade $(\mathrm{F}=$ 
Tabela 2. Relação professor-aluno a partir de indicadores de conflito e afinidade.

\begin{tabular}{|c|c|c|c|c|c|c|c|c|}
\hline $\begin{array}{l}\text { Sexo dos } \\
\text { Alunos }\end{array}$ & Indicadores & $\mathrm{N}$ & Mín. & Máx. & $M$ & D P & $F$ & $p$ \\
\hline \multirow[t]{2}{*}{ Feminino } & & 53 & 16 & 33 & 23,23 & 4,95 & & \\
\hline & Afinidade & & & & & & & \\
\hline Masculino & & 47 & 13 & 33 & 20,21 & 5,50 & & \\
\hline \multirow[t]{2}{*}{ Feminino } & & 53 & 8 & 29 & 17,57 & 4,94 & & \\
\hline & Conflito & & & & & & & \\
\hline \multirow[t]{2}{*}{ Masculino } & & 47 & 9 & 25 & 15,68 & 3,23 & & \\
\hline & Afinidade & & 13 & 33 & 21,81 & 5,41 & 12,19 & 0,001 \\
\hline \multirow[t]{2}{*}{$\begin{array}{l}\text { Feminino e } \\
\text { Masculino }\end{array}$} & & 100 & & & & & & \\
\hline & Conflito & & 8 & 29 & 16,68 & 4,31 & 1,99 & 0,162 \\
\hline
\end{tabular}

Nota. $\mathrm{p} \leq 0,01$.

Tabela 3. Relação dos indicadores, conforme o Desempenho Acadêmico dos alunos.

\begin{tabular}{|c|c|c|c|c|c|}
\hline Desempenho Acadêmico & $\mathrm{N}$ & & $\begin{array}{c}\text { Coeficiente Correlação Nível } \\
\text { Significância }\end{array}$ & Afinidade & Conflito \\
\hline \multirow{2}{*}{ Português } & & $r$ & & 0,16 & 0,08 \\
\hline & & $p$ & & 0,124 & 0,405 \\
\hline \multirow{2}{*}{ Física } & & & $r$ & 0,10 & 0,08 \\
\hline & & & $p$ & 0,336 & 0,410 \\
\hline \multirow{2}{*}{ Filosofia } & & & $r$ & 0,17 & 0,20 \\
\hline & & & $p$ & 0,098 & 0,047 \\
\hline \multirow{2}{*}{ Sociologia } & & & $r$ & 0,13 & $-0,00$ \\
\hline & 100 & & $p$ & 0,204 & 0,977 \\
\hline \multirow{2}{*}{ Geografia } & & & $r$ & 0,27 & $-0,03$ \\
\hline & & & $p$ & 0,006 & 0,761 \\
\hline \multirow{2}{*}{ Educação Física } & & & $r$ & $-0,17$ & $-0,12$ \\
\hline & & & $p$ & 0,092 & 0,226 \\
\hline \multirow{2}{*}{ História } & & & $r$ & $-0,04$ & $-0,02$ \\
\hline & & & $\mathrm{p}$ & 0,665 & 0,856 \\
\hline
\end{tabular}

Nota.Correlação é significativa ao nível de 0,05. Correlação é significativa ao nível de 0,01. 
$12,19 ; p=0,001)$. Estes valores são corroborados pelo estudo de (Barbosa, Campos, \& Valentim, 2011), ao evidenciar que a relação professor-aluno é mais conflituosa quando se trata de discentes do sexo masculino. Desta maneira, a afinidade é maior com as alunas.

Deve-se ressaltar que na pesquisa de Barbosa e cols. (2011), a amostragem foi efetuada com 21 professoras que lecionavam em salas do primeiro ao quinto ano do ensino fundamental, diferente do presente estudo.

Ao correlacionar as variáveis de desempenho acadêmico com os indicadores de afinidade e conflito verificaram se que existe correlação positiva entre o indicador de conflito e o desempenho acadêmico da disciplina Filosofia, mesmo a correlação sendo fraca ela possui significância $(r=0,20$; $p=0,047$ ), pois o aumento do conflito é correspondido pelo acréscimo do desempenho acadêmico dos alunos; isso pode ser explicado por se tratar de uma disciplina onde o debate com os discentes é estimulado, pois o ensino de Filosofia é uma pedagogia comunicativa, os sujeitos ampliam a sua consciência crítica, como atores diretos da interação social em sala de aula (Tesser, Horn, \& Junkes, 2012).

Já na disciplina de Geografia, existe também uma correlação positiva fraca com o indicador de afinidade, apresentando significância $(r=0,27 ; p=0,006)$, onde o aumento de afinidade é correspondido pelo aumento do desempenho acadêmico, ou seja, quanto melhor for o relacionamento do professor com o aluno, maior será a nota do discente.

Contudo, uma forma para se explicar a correlação fraca pode ser resultado de serem respondidos por diferentes participantes. Pesquisas relatam que a correlação entre os diferentes participantes geralmente é baixa ou moderada, pois a opinião fornecida é influenciada por expectativas pessoais, valores e crenças, onde os adultos (professores) podem emitir informações enviesadas, visto que os alunos são sempre comparados com outros, ou avaliados segundo os valores e expectativas dos respondentes (Petrucci \& cols., 2014).

Para Barbosa e cols. (2011), as percepções sobre a qualidade do relacionamento entre docente e alunado se encontram na adequação com o desempenho das práticas acadêmicas, pois estão relacionadas à compreensão do professor, que fornece informações que melhoram o entendimento do relacionamento que se mantém com o alunado no seu cotidiano no ambiente escolar.

O aumento da idade do discente corresponde a um acréscimo de afinidade $(r=0,25 ; p=0,013)$; a magnitude do coeficiente baixa indica que esta relação não acontece em todos os casos, apesar de ser significativa. Outra abordagem que pode ser considerada é que quanto maior a idade desse aluno, maior será a sua afinidade.

Esse resultado não foi encontrado no estudo de Barbosa e cols. (2011), pesquisa em que as associações na relação professor-aluno tendem a ser mais negativas com estudantes mais velhos, ou seja, mais conflituosas e menos afetivas.

Novamente, vale ressaltar que a amostragem da pesquisa de Barbosa e cols. (2011) foi com 21 professoras que lecionavam em salas do primeiro ao quinto ano do ensino fundamental, e o presente estudo foi feito com 10 professores de ambos os sexos e alunos do segundo ano do ensino médio.

O resultado apresentado na tabela sugere que garotas e rapazes tenderam a ser avaliados da mesma forma pelos seus professores (Média do Total= 16,71 e 16,68). Contudo, a análise descritiva pontuou que as meninas foram melhor avaliadas pelas professoras (Média: $\mathrm{F}=26,25$ e $\mathrm{M}=$ $22,34)$, porém não houve diferença no indicador de conflito (Média: $\mathrm{F}=16,50$ e $\mathrm{M}=17,88$ ).

Sendo assim, o gênero dos professores foi uma variável que influenciou a afinidade ( $F=4,49$ e $p=0,037)$, apontando a existência de afinidade no relacionamento professor-aluno no que se refere ao sexo feminino dos docentes.

$\mathrm{Na}$ literatura pesquisada no que se refere à variável sexo, mesmo por se tratar de variáveis com recortes de idades distintos, existem evidências de que as relações entre professoras e as garotas são mais harmoniosas, pois as alunas se atentam mais ao meio social e diversificam mais

Tabela 4. Relação dos indicadores, de acordo com a idade dos alunos.

\begin{tabular}{|c|c|c|c|c|}
\hline Variável & $\mathrm{N}$ & $\begin{array}{l}\text { Coeficiente Correlação e } \\
\text { Nível Significância }\end{array}$ & Afinidade & Conflito \\
\hline \multirow{3}{*}{ Idade do Aluno } & & $r$ & 0,25 & 0,00 \\
\hline & 100 & & & \\
\hline & & $p$ & 0,013 & 0,993 \\
\hline
\end{tabular}

Nota. $\mathrm{p} \leq 0,05$. 
Tabela 5. Relação dos indicadores com o Sexo dos Alunos e dos Professores.

\begin{tabular}{|c|c|c|c|c|c|c|c|}
\hline $\begin{array}{l}\text { Sexo } \\
\text { Alunos }\end{array}$ & $\begin{array}{c}\text { Sexo } \\
\text { Professores }\end{array}$ & Indicadores & Média & $\begin{array}{l}\text { Desvio } \\
\text { Padrão }\end{array}$ & $\mathrm{N}$ & $\mathrm{F}$ & $p$ \\
\hline & $\mathrm{F}$ & & 26,25 & 3,82 & 12 & & \\
\hline \multirow[t]{4}{*}{$\mathrm{F}$} & M & & 22,34 & 4,93 & 41 & & \\
\hline & Total & & 23,23 & 4,95 & 53 & & \\
\hline & & & & & & 4,49 & 0,037 \\
\hline & $\mathrm{F}$ & & 20,78 & 4,65 & 18 & & \\
\hline \multirow[t]{3}{*}{$M$} & M & Afinidade & 19,86 & 6,03 & 29 & & \\
\hline & Total & & 20,21 & 5,51 & 47 & & \\
\hline & $\mathrm{F}$ & & 22,97 & 5,06 & 30 & & \\
\hline \multirow[t]{3}{*}{ Total } & $M$ & & 21,31 & 5,51 & 70 & & \\
\hline & Total & & 21,81 & 5,41 & 100 & & \\
\hline & $\mathrm{F}$ & & 16,50 & 6,30 & 12 & & \\
\hline \multirow[t]{4}{*}{$\mathrm{F}$} & M & & 17,88 & 4,52 & 41 & & \\
\hline & Total & & 17,57 & 4,94 & 53 & & \\
\hline & & & & & & 0,01 & 0,907 \\
\hline & $\mathrm{F}$ & & 16,67 & 3,71 & 18 & & \\
\hline \multirow[t]{3}{*}{$M$} & M & Conflito & 15,07 & 2,79 & 29 & & \\
\hline & Total & & 15,68 & 3,23 & 47 & & \\
\hline & $\mathrm{F}$ & & 16,60 & 4,81 & 30 & & \\
\hline \multirow[t]{2}{*}{ Total } & $M$ & & 16,71 & 4,12 & 70 & & \\
\hline & Total & & 16,68 & 4,31 & 100 & & \\
\hline
\end{tabular}

Nota. $p \leq 0,05$.

seus relacionamentos (Barbosa \& cols., 2011; Petrucci \& cols., 2014).

No estudo de Petrucci e cols. (2014), os autores argumentam que mesmo sendo do sexo feminino ou masculino, os docentes indicaram maior nível de conflito no relacionamento com os meninos do que com as meninas, isso pode ser esclarecido por uma maior valorização de particularidades femininas na escola como: conformidade, cooperação e atenção em contraposição a aspectos masculinos como: diligência, impulsividade e autonomia.

Contudo, neste estudo, ao se verificar as médias é improvável relatar a existência de conflito, vale salientar que o nível de significância para conflito não foi representativo $(p=0,907)$. Nesse caso, é preciso examinar com atenção os resultados de análises comparativas entre características dos alunos, com estudos de invariância da Escala da Relação Professor Aluno entre sexo e idade dos alunos para a 
avaliação da medida entre diferentes grupos, mas este não foi o propósito da presente pesquisa, mas de acordo com os autores, esses indicadores de variabilidade não demonstram implicações para as qualidades psicométricas (Koomen, Verschueren, Schooten, Jak, \& Pianta, 2012).

\section{Considerações Finais}

Neste estudo ocorreu a corroboração parcialmente da hipótese, conforme apontado pela literatura de Barbosa e cols.(2011) e Petrucci e cols.(2014). Dessa maneira, a análise do relacionamento dos professores com os alunos a partir de indicadores de conflito e afinidade está associada à variável desempenho acadêmico, envolvendo sete disciplinas, somente duas apresentaram resultados satisfatórios conforme os valores estatísticos, sendo essa uma das contribuições deste estudo. Com isso, são necessários estudos que contribuam com essa limitação, pois se trata de uma amostragem diferente das pesquisas já realizadas.

No que se refere à interação dos indicadores com a idade dos alunos, foram encontrados resultados satisfatórios estatisticamente, mas por se tratar de um tipo de amostragem diferente dos outros estudos, não foi possível um embasamento teórico para comprovar as descobertas, todavia esta pesquisa já está contribuindo com novas evidências. Futuramente um maior aprimoramento da quantidade de participantes e de mais de um lugar para a coleta de dados poderá ser realizado por futuros pesquisadores.

No caso das variáveis sexo dos alunos e sexo dos respondentes foram alcançados resultados adequados aos estudos existentes, pois mesmo as amostras tendo perfis de idade diferente, se assemelham às descobertas das literaturas de Barbosa e cols. (2011) e Petrucci e cols.(2014). Assim, se fazem necessário outros estudos preliminares conforme apontam Koomen e cols. (2012).

Uma das principais contribuições da presente pesquisa foi ir além desse recorte amostral comumente utilizado, que está agregado aos indicadores das interações entre os principais protagonistas do ambiente escolar: docente e discente. Recomendam-se, dessa forma, pesquisas que associem as variáveis aqui estudadas na relação professor-aluno, como: sexo e idade de ambos os participantes e desempenho acadêmico de todas as matérias estudadas no currículo escolar.

Outras particularidades que podem influenciar as condutas externas e podem posteriormente compor a base de novas pesquisas são as condutas que envolvem tratativas negativas como: faltar com a verdade, roubar, agredir, gritar, locomover-se o tempo todo, falar ininterruptamente, variáveis que compõem um desafio diário para os professores, sendo esses tipos de comportamentos que colocam em risco a qualidade do relacionamento com o docente e se associam a uma convivência negativa entre o professor e o aluno.

Apesar das limitações deste estudo foram encontrados alguns resultados discrepantes e também resultados que corroboram aqueles apresentados nas demais pesquisas.

Entre as limitações, destaca-se o pequeno tamanho da amostra, a execução da coleta de dados efetuada em uma única instituição de ensino, a não realização de análises de invariância com a ERPA, somente a percepção do professor para a avaliação dessa relação e a dificuldade de se encontrar outra medida brasileira que mensure o clima escolar.

Compreendendo esse contexto, os resultados encontrados nesta pesquisa serão capazes de estender a compreensão da relação professor-aluno para o desenvolvimento dos discentes, envolvendo os indicadores de conflito e afinidade dessa medida para o contexto brasileiro.

Além disso, será possível a consulta da dissertação que gerou este artigo para auxiliar na consulta e análise da realização de novos estudos, que deverão ser conduzidos para complementar as demais pesquisas no âmbito empírico, na realização de coleta de dados em mais de uma escola, de uma maior amplitude da amostra do público adolescente em função de suas características sociais e/ou individuais.

\section{Referências}

Barbosa, A. J. G., Campos, R. A., \& Valentim, T. A.(2011). A diversidade em sala de aula e a relação professor-aluno. Estudos de Psicologia, 28(4), 453-461. Recuperado: 15 dez. 2015. Disponível: http://www. scielo.br/scielo.php?script=sci_arttext\&pid=S0103.

Berry, D. \& O'Connor, E. (2010). Behavioral risk, teacher-child relationships, and social skill development across middle childhood: A child-by-environment analysis of change. Journal of Applied Developmental Psychology, 31, 1-14.

Crosnoe, R., Johnson, M. K., \& Elder, G. H. (2004). Intergenerational bonding in school: The behavioral and contextual correlates of student-teacher relationships. Sociology of Education, 77, 60-81.

Hamre, B. K. \& Pianta, R. C. (2006). Student-teacher relationships. Em G. G. Bear \& K. M. Minke (Orgs.), Children's needs III: Development, prevention, and intervention (pp. 59-72). Washington, D. C.: National Association of School Psychologists.

Koomen, H. M. Y., Verschueren, K., Van Shooten, E., Jak, S., \& Pianta, R. C. (2012). Validating the Student-Teacher Relationship Scale: Testing factor structure and measurement invariance across child gender and age in a Dutch sample. Journal of School Psychology, 50, 215-234.

Maldonado-Carreño, C. \& Votruba-Drzal, E. (2011). Teacher-child relationships and the development of academic and behavioral skills during elementary school: A within- and between-child analysis. Child Development, 82, 601-616.

Myers, S. S. \& Pianta, R. C. (2008). Developmental commentary: Individual and contextual influences on student-teacher 
relationships and children's early problem behaviors. Journal of Clinical Child \& Adolescent Psychology, 37, 600-608.

O'Connor, E. E., Collins, B. A., \& Supplee, L. (2012). Behavior problems in late childhood: The roles of early maternal attachment and teacher-child relationship trajectories. Attachment \& Human Development, 14, 265-288.

Petrucci, G. W., Borsa, J. C., Barbosa, A. J. G., \& Koller, S. H. (2014). Adaptação cultural e evidências de validade da Escala de Relacionamento Professor-Aluno. Avaliação Psicológica, 13(1), 133-142. Recuperado: 15 dez. 2015. Disponível: http://pepsic. bvsalud.org/scielo.php.

Pianta, R. C. (1992). Student-Teacher Relationship Scale. Unpublished measure. Viginia: University of Virginia. Recuperado: 15 dez. 2015. Disponível: http://www.virginia.edu/vpr/CASTL/files/ Student-TeacherRelationshipScale.pdf

Pianta, R. C. (1999). Enhancing relationships between children and teachers. Washington, DC: American Psychological Association.

Pianta, R. C. \& Allen, J. P. (2008). Building capacity for positive youth development in secondary school classrooms: Changing teachers' interactions with students. Em M. B. Shinn \& H. Yoshikawa (Orgs.), Toward positive youth development: Transforming schools and community programs (pp. 21-40). New York, NY: Oxford University Press.

Pianta, R. C. \& Stuhlman, M. W. (2004). Conceptualizing risk in relational terms: Associations among the quality of child-adult relationships prior to school entry and children's developmental outcomes in first grade. Educational and Child Psychology, 21, 3245.

Silver, R. B., Measelle, J. R., Armstrong, J. M., \& Essex, M. J. (2005). Trajectories of classroom externalizing behavior: Contributions of child characteristics, family characteristics, and the teacherchild relationship during the school transition. Journal of School Psychology, 43, 39-60.

Tesser, G. J., Horn, G. B., \& Junkes, D. (2012). A Filosofia e seu ensino a partir de uma perspectiva da teoria crítica. Educar em revista, (46), 113-126. Recuperado: 04 nov. 2015. Disponível: http://www.scielo.br/scielo.php?script=sci_arttext\&pid=S010440 602012000400009\&lng=en\&nrm=iso.http://dx.doi.org/10.1590/ S0104-40602012000400009.

\section{Sobre os autores}

Victor Silva Ferreira (vsjfdd@yahoo.com.br)

Professor nas Faculdades Integradas Campos Sales na área de Administração de Empresas.

Márcia Siqueira de Andrade (mandrade@unifieo.br)

Doutora em Psicologia da Educação pela Pontifícia Universidade Católica de São Paulo, Coordenadora do Programa de Psicologia Educacional do UNIFIEO (Mestrado e Doutorado). 